\title{
Cafe-au-lait spots as a clinical sign of syndromes
}

\author{
Manchas café-com-leite como um sinal clínico de síndromes \\ Manchas café con leche como signo clínico de síndromes
}

Received: 06/27/2021 | Reviewed: 07/04/2021 | Accept: 07/12/2021 | Published: 07/23/2021

\author{
Adriana Amaral Carvalho \\ ORCID: https://orcid.org/0000-0003-4733-6209 \\ State University of Montes Claros, Brazil \\ E-mail: adrianaamaral.carvalho@gmail.com \\ Daniella Reis Barbosa Martelli \\ ORCID: https://orcid.org/0000-0002-3979-7497 \\ State University of Montes Claros, Brazil \\ E-mail: daniellareismartelli@yahoo.com.br \\ Maria Fernanda Amaral Carvalho \\ ORCID: https://orcid.org/0000-0001-8789-2387 \\ Medical Science College-MG, Brazil \\ E-mail: nandaamaralc31@gmail.com \\ Mário Sérgio Oliveira Swerts \\ ORCID: https://orcid.org/0000-0003-1867-7880 \\ University of Alfenas, Brazil \\ E-mail: mariosergio.swerts@unifenas.br \\ Hercílio Martelli Júnior \\ ORCID: https://orcid.org/0000-0001-9691-2802 \\ State University of Montes Claros, Brazil \\ E-mail: hmjunior2000@yahoo.com
}

\begin{abstract}
Several studies describe the frequent association of cafe-au-lait spots with neurofibromatosis. However, many other genetic diseases might be associated with the presence of café-au-lait spots. Several genetic diseases are rare. In most cases, syndromes present themselves as a set of signs and symptoms that may present varied penetrance, therefore largely reducing the percentage of final diagnosis. Exploration of clinical symptomatology is essential for the understanding and diagnosis of syndromes. In this review, we conduct an extensive literature search looking for research that investigated diseases that may be present simultaneously with the cafe-au-lait spots. A total of 60 genetic diseases were found, all of them rare. These syndromes were evaluated based on their most relevant features and described in a summary of the typical, general, and head and neck findings. The available OMIM number, mode of inheritance, chromosome, mutated genes, and affected proteins were also listed. The considerable variety of diseases associated with the presence of cafe-au-lait spots and the fact that many of these conditions affect various organ systems with diverse phenotypic presentations is a diagnostic and therapeutic challenge. The objective of this study was to provide health professionals with an instrument containing a broad spectrum of genetic diseases coincident with the presence of cafe-au-lait spots in order to facilitate the differential and final diagnosis of these syndromes.
\end{abstract}

Keywords: Cafe-au-lait spots; Hyperpigmentation; Neurofibromatosis; Inborn genetic diseases; Hereditary syndromes.

\section{Resumo}

Vários estudos descrevem a associação frequente de manchas café-com-leite com neurofibromatose. No entanto, muitas outras doenças genéticas podem estar associadas à presença de manchas café-com-leite. Várias doenças genéticas são raras. Na maioria dos casos, as síndromes se apresentam como um conjunto de sinais e sintomas que podem apresentar penetrância variada, reduzindo bastante o percentual de diagnóstico final. A exploração da sintomatologia clínica é essencial para a compreensão e diagnóstico das síndromes. Nesta revisão, realizamos uma extensa investigação bibliográfica em busca de pesquisas que avaliaram doenças que podem estar presentes simultaneamente com as manchas café-com-leite. Um total de 60 doenças genéticas foram encontradas, todas raras. Essas síndromes foram avaliadas com base em suas características mais relevantes e descritas em um sumário contendo os achados típicos, gerais e da cabeça e pescoço. O número OMIM disponível, modo de herança, cromossomo, genes mutados e proteínas afetadas também foram listados. A considerável variedade de doenças associadas à presença de manchas café-com-leite e o fato de que muitas dessas condições afetam vários sistemas orgânicos com diversas apresentações fenotípicas é um desafio diagnóstico e terapêutico. O objetivo deste estudo foi fornecer aos profissionais de saúde um instrumento contendo um amplo espectro de doenças genéticas associadas à presença de manchas café-com-leite, a fim de facilitar o diagnóstico diferencial e final dessas síndromes. 
Palavras-chave: Manchas café-com-leite; Hiperpigmentação; Neurofibromatose; Doenças genéticas inatas; Síndromes hereditárias.

\section{Resumen}

Varios estudios describen la asociación frecuente de manchas café con leche con neurofibromatosis. Sin embargo, muchas otras enfermedades genéticas pueden estar asociadas con la presencia de manchas café con leche. Varias enfermedades genéticas son raras. En la mayoría de los casos, los síndromes se presentan como un conjunto de signos y síntomas que pueden presentar una penetrancia variada, reduciendo en gran medida el porcentaje de diagnóstico final. La exploración de la sintomatología clínica es fundamental para la comprensión y el diagnóstico de los síndromes. En esta revisión, realizamos una extensa investigación bibliográfica en busca de trabajos que evaluaran enfermedades que pueden estar presentes simultáneamente con manchas café con leche. Se encontraron un total de 60 enfermedades genéticas, todas raras. Estos síndromes se evaluaron en función de sus características más relevantes y se describieron en un resumen de los hallazgos típicos, generales y de cabeza y cuello. También se enumeraron el número OMIM disponible, el modo de herencia, el cromosoma, los genes mutados y las proteínas afectadas. La considerable variedad de enfermedades asociadas con la presencia de manchas café con leche y el hecho de que muchas de estas condiciones afectan a varios sistemas de órganos con diversas presentaciones fenotípicas es un desafío diagnóstico y terapéutico. El objetivo de este estudio fue proporcionar a los profesionales de la salud un instrumento que contenga un amplio espectro de enfermedades genéticas coincidentes con la presencia de manchas café con leche, para facilitar el diagnóstico diferencial y final de estos síndromes.

Palabras clave: Manchas de café con leche; Hiperpigmentación; Neurofibromatosis; Enfermedades genéticas innatas; Síndromes hereditarios.

\section{Introduction}

Cafe-au-lait spots (CALS) or cafe-au-lait macules (CALM) are characteristically well-defined lesions, with a homogeneous light brown or medium to dark brown spots in dark-skinned people, that might be found all over the body except for the scalp, palms and soles (Hamm, Emmerich \& Olk, 2019). Morphologically, they have been described as oval-shaped and with smooth edges or irregular contours, ranging in size from $0.2 \mathrm{~cm}$ to $30 \mathrm{~cm}$ in diameter, being smaller in young children since they increase proportionally to the body surface (Fistarol \& Itin, 2010; Shah, 2010; Hamm et al., 2019). Histologically, they present increased melanin content in both melanocytes and basal keratinocytes, with giant melanosomes also being observed (Shah, 2010). They should be distinguished from lentigo (small pigmented spots with clearly defined edges, varying in size from 2 to $20 \mathrm{~mm}$, usually smaller than $1 \mathrm{~cm}$, that might occur anywhere on the skin) and nevus (a congenital or acquired usually highly pigmented area on the skin, flat or raised), clinical entities that alone will not be analyzed in this study (Fistarol \& Itin, 2010).

CALM may be present at birth or develop in the first years of life, which occurs in most cases (Hamm et al, 2019).

Isolated CALM is a common finding (10-36\% of healthy people) with no clinical significance when dissociated from other findings (Rivers et al., 1985; Hamm et al, 2019). However, the presence of multiple CALMs, large segmental CALM, other cutaneous anomalies, associated facial dysmorphism or unusual findings on physical examination, may suggest the possibility of an associated genetic disease and should be promptly investigated (Shah, 2010).

Several steps are involved in determining skin color, such as lineage specification from embryonic neural crest cells (melanoblasts), melanoblast migration to skin of the embryo; proliferation and survival of the melanocytes in the basal layer of the epidermis; biogenesis of the melanosomes in the melanocytes; production of melanin granules in the melanosomes; translocation of melanosomes from the perinuclear region to the peripheral region of the melanocytes; transfer of the melanosomes from the melanocytes to the keratinocytes; and translocation of the transferred melanin granules from the peripheral region to the supranuclear region of the keratinocytes (Cichorek, Wachulska, Stasiewicz \& Tymińska, 2013; Oiso, Fukai, Kawada \& Suzuki, 2013). In parallel, a complex melanogenic paracrine network between the mesenchymal and epithelial cells regulates the processes involved in determining skin color after birth (Picardo \& Cardinali, 2011; Oiso et al., 2013).

Multiple genes encode component proteins or signaling pathway regulators that control these paracrine network and the melanogenic growth factors which play a crucial role in the control of physiological and pathological skin pigmentation (Picardo 
\& Cardinali, 2011). In this category are the KITLG gene, which encodes the Kit ligand (ligand for the receptor-type proteintyrosine kinase KIT) and the proto-oncogene $c$-KIT encoding the receptor tyrosine-protein kinase KIT, which activate the Ras/mitogen-activated protein kinase (RAS/MAPK) signaling pathway (Picardo \& Cardinali, 2011; Oiso et al., 2013; Zhang, Li \& Yao, 2016). Kit signaling plays an important role in a variety of physiological processes that occur in many cell types in the body, such as hematopoietic stem cells, mast cells, melanocytes, and germ cells (Ronnstrand, 2004). The RAS family comprises genes expressed in several types of normal cells: $H-R A S, N-R A S$, and $K-R A S$, which plays an important role in intracellular signaling pathways and in the regulation of functions such as cell cycle control, differentiation, growth, and cell senescence (Boquett \& Ferreira, 2010). A large group of diseases associated with CALM result from germline mutations in these associated genes.

This article provides a critical review of the literature on genetic syndromes associated with CALM. In addition, the genotypic and phenotypic alterations of the identified diseases are described.

\section{Methodology}

We conducted a bibliographical research from September 2020 to January 2021, looking for studies that investigated diseases that can present CALM as a clinical manifestation. The compendium of human genes and genetic diseases OMIM (Online Mendelian Inheritance in Man) was used as the main source of data for the identification of associated syndromes. The descriptors used for research were: "cafe-au-lait spots", "hyperpigmentation", "neurofibromatosis", "inborn genetic diseases" and "hereditary syndromes".

Through a critical analysis of the literature, this narrative review was designed to provide a comprehensive understanding of the syndromes associated with CALM, in order to contribute to health professionals, facilitating the differential and final diagnosis of these syndromes. These objectives are in alignment with the reported by Oates \& Harris (2015) on the importance of this scientific methodology, either to inform practice and to provide a comprehensive understanding of what is known about a topic.

The genotypic and phenotypic manifestations, as well as the dysmorphic changes in the head and neck of the identified syndromes, were obtained from the OMIM and Orphanet (Online database of rare diseases and orphan drugs) databases, and the book Gorlin's Syndromes of the Head and Neck (Hennekam, Allanson, Krantz, \& Gorlin, 2010). Then, the search was expanded to other databases, such as PubMed (https://pubmed.ncbi.nlm.nih.gov/) and Virtual Health Library (VHL) (www.bvsalud.org). No limits were established regarding the date of the published works.

\section{Results}

Several genetic syndromes can be associated with CALM, all of them rare in occurrence. In the research conducted at OMIM, a total of 60 associated genetic diseases were identified. These syndromes were classified into subsections based on their most relevant features and described in a summary of typical, general, and head and neck findings (Table 1). Among the 60 diseases identified, 40 of them had frequent and significant changes in pigmentation or skin formation. Multiple CALMs are part of the clinical manifestation of at least 29 of these syndromes. In most other syndromes, the presence of CALM was simply an occasional finding, with a total number of affected patients too small to establish any overall rate of involvement, or its presence is not relevant compared to other more evident clinical characteristics. 
Table 1. Genetic syndromes reported with cafe-au-lait spots: features (classified according to the most relevant changes).

\begin{tabular}{|c|c|}
\hline Highlighted changes: skin & Others features \\
\hline Neurofibromatosis type I & $\begin{array}{l}\text { Typical: multiple CALMs; Lisch nodules; cutaneous/subcutaneous/plexiform neurofibromas; axillary } \\
\text { and inguinal freckling. } \\
\text { General: mental retardation (mild), hydrocephalus, learning disabilities; renal artery stenosis, } \\
\text { hypertension; spina bifida, scoliosis, pseudoarthrosis, local bone overgrowth; hypothalamic tumor, } \\
\text { neurofibrosarcoma, rhabdomyosarcoma, duodenal carcinoid, somatostatinoma, pheochromocytoma, } \\
\text { astrocytoma, meningioma, parathyroid adenoma, malignant peripheral nerve and central nervous system } \\
\text { tumors. } \\
H \& N \text { : macrocephaly, sphenoid dysplasia; hypertelorism, choroidal spots, optic glioma. }\end{array}$ \\
\hline $\begin{array}{l}\text { Neurofibromatosis type } \\
\text { II }\end{array}$ & $\begin{array}{l}\text { Typical: multiple neoplasia syndrome: tumors in the eighth cranial nerve }(90 \%) \text {, meningiomas, } \\
\text { schwannomas. } \\
\text { General: CALM ( } 40 \%) \text {, neurofibroma; ataxia, peripheral neuropathy; glioma, ependymoma, } \\
\text { astrocytoma, vestibular schwannoma. }\end{array}$ \\
\hline
\end{tabular}

Ref.

OMIM (162200)

$\boldsymbol{H} \& \boldsymbol{N}$ : hearing loss; lenticular opacities, retinal hamartoma

OMIM (101000)

Plana-Pla, 2017

\begin{tabular}{|c|c|c|}
\hline Highlighted changes: skin & Others features & Ref. \\
\hline Legius syndrome & $\begin{array}{l}\text { Typical: multiple CALMs, variable dysmorphic features, lipomas, mild learning disabilities. } \\
\text { General: freckling; deficit attention; pectus deformities; hypotonia. } \\
\text { H\&N: macrocephaly; triangular face; short neck, low-set ears; hypertelorism, downslanting palpebral } \\
\text { fissures, epicanthal folds, ptosis; micrognathia; low-posterior hairline. }\end{array}$ & OMIM (611431) \\
\hline $\begin{array}{l}\text { Multiple cafe-au-lait } \\
\text { spots }\end{array}$ & $\begin{array}{l}\text { Typical: multiple CALMs without other neurofibromatosis changes. } \\
\text { General: CALMs. } \\
\text { H\&N: no specific changes. }\end{array}$ & $\begin{array}{l}\text { OMIM (114030) } \\
\text { Madson, } 2012\end{array}$ \\
\hline $\begin{array}{l}\text { McCune-Albright } \\
\text { syndrome }\end{array}$ & $\begin{array}{l}\text { Typical: polyostotic fibrous dysplasia, CALM, precocious puberty. } \\
\text { General: gastrointestinal polyps; pathologic fracture; hyperthyroidism, hyperparathyroidism, Cushing } \\
\text { syndrome, hyperprolactinemia, acromegaly. } \\
\text { H\&N: craniofacial hyperostosis, facial asymmetry, deafness, blindness; pituitary adenoma. }\end{array}$ & $\begin{array}{l}\text { OMIM (174800) } \\
\text { Hamm, } 2019\end{array}$ \\
\hline $\begin{array}{l}\text { Gastrocutaneous } \\
\text { syndrome }\end{array}$ & $\begin{array}{l}\text { Typical: peptic ulcer, hiatal hernia and multiple lentigines/CALMs. } \\
\text { General: multiple lentigines/CALMs. } \\
\text { H\&N: hypertelorism, myopia. }\end{array}$ & $\begin{array}{l}\text { OMIM (137270) } \\
\text { Hamm,2019 } \\
\text { Halal, } 1982\end{array}$ \\
\hline $\begin{array}{l}\text { Familial progressive } \\
\text { hyperpigmentation }\end{array}$ & $\begin{array}{l}\text { Typical: patches of hyperpigmentation in the skin. } \\
\text { General: hyperpigmentation - present as diffuse hyperpigmentation, or as dots, streaks, patches, whorls; } \\
\text { CALM. } \\
\text { H\&N: no specific changes. }\end{array}$ & OMIM (614233) \\
\hline $\begin{array}{l}\text { Familial progressive } \\
\text { hyperpigmentation and } \\
\text { hypopigmentation }\end{array}$ & $\begin{array}{l}\text { Typical: diffuse hyperpigmentation and larger hypopigmented ash-leaf macules. } \\
\text { General: progressive hyperpigmentation (trunk, limbs, palms, soles) and hypopigmentation, multiple } \\
\text { CALMs, lentigines, vitiligo (rare); hyperkeratosis. } \\
\text { H\&N: hyperpigmented patches (face, neck, oral mucosa). }\end{array}$ & $\begin{array}{l}\text { OMIM (145250) } \\
\text { Zeng, } 2016 \\
\text { Amyere, } 2011\end{array}$ \\
\hline $\begin{array}{l}\text { Adams-Oliver syndrome } \\
4\end{array}$ & $\begin{array}{l}\text { Typical: aplasia cutis and terminal transverse limb defects. } \\
\text { General: cutis marmorata, CALM (rare); dysplastic/aplastic toenails; temporal/occipital infarct; heart } \\
\text { defect; umbilical hernia; digital defects. } \\
\text { H\&N: cutis aplasia and bony defect (scalp). }\end{array}$ & OMIM (615297) \\
\hline $\begin{array}{l}\text { Piebald trait } \\
\text { (piebaldism) }\end{array}$ & $\begin{array}{l}\text { Typical: depigmented patches: skin and hair; CALM and freckling - not usual features. } \\
\text { General: white forelock; absent pigmentation: forehead, eyebrows, chin, chest, abdomen, limbs; } \\
\text { Hirschsprung disease; frequent epitheliomas. } \\
\text { H\&N: occasional deafness; heterochromia iridis. }\end{array}$ & $\begin{array}{l}\text { OMIM (172800) } \\
\text { Hamm, } 2019 \\
\text { Oiso, } 2013 \\
\text { Chiu, } 2013\end{array}$ \\
\hline Cowden syndrome 1 & $\begin{array}{l}\text { Typical: macrocephaly, acral keratoses, facial trichilemmomas, papillomatous papules, increased risk } \\
\text { for carcinoma. } \\
\text { General: CALM-penis, body; multiple skin tags; multiple hamartomas; vascular anomalies; pectus } \\
\text { excavatum; vaginal/vulvar/ovarian cysts; gynecomastia; gastrointestinal polyps, colonic diverticulosis; } \\
\text { seizure, Lhermitte-Duclos disease, mental retardation (12\%); meningioma, neuromas. } \\
\text { H\&N: 'Birdlike' facies; hearing loss; cataract, angioid streak; microstomia, oral papillomas, scrotal } \\
\text { tongue. }\end{array}$ & $\begin{array}{l}\text { OMIM (158350) } \\
\text { Hamm, } 2019 \\
\text { Guimarães,2002 }\end{array}$ \\
\hline $\begin{array}{l}\text { Peutz-Jeghers } \\
\text { syndrome }\end{array}$ & $\begin{array}{l}\text { Typical: melanocytic macules: lips, buccal mucosa, digits; multiple gastrointestinal polyps; increased } \\
\text { risk of neoplasms. } \\
\text { General: hyperpigmented spots; CALM (unusual); bronchial polyps; biliary tract polyps; } \\
\text { hamartomatous polyps (stomach to rectum); ovarian cysts; ureteral polyps, bladder polyps; clubbing of } \\
\text { fingers; precocious puberty; gastrointestinal carcinoma, breast cancer, thyroid cancer, } \\
\text { lung/pancreatic/uterine/ovarian cancers. } \\
H \& N \text { : nasal polyps. }\end{array}$ & $\begin{array}{l}\text { OMIM (175200) } \\
\text { Plataras, } 2018\end{array}$ \\
\hline Carney complex & $\begin{array}{l}\text { Typical: multiple neoplasia syndrome: cardiac, endocrine, cutaneous, neural myxomatous tumors; } \\
\text { pigmented lesions of the skin and mucosae. } \\
\text { General: lentigines; blue nevi/other nevi, CALM; schwannoma; pigmented adrenal dysplasia, Cushing } \\
\text { disease, acromegaly, thyroid hyperplasia; adrenocortical hyperplasia, Sertoli cell tumor, pituitary } \\
\text { adenoma, mammary fibroadenoma, thyroid carcinoma, pheochromocytoma; atrial/ventricular myxoma. } \\
\text { H\&N: conjunctival pigmentation, eyelid myxoma; hirsutism, red hair. }\end{array}$ & $\begin{array}{l}\text { OMIM (160980) } \\
\text { Hamm, } 2019\end{array}$ \\
\hline
\end{tabular}


Research, Society and Development, v. 10, n. 9, e14310917607, 2021

(CC BY 4.0) | ISSN 2525-3409 | DOI: http://dx.doi.org/10.33448/rsd-v10i9.17607

Highlighted changes: skin and short stature

Leopard syndrome $1 \quad$ Typical: multiple lentigines, electrocardiographic abnormalities, hypertelorism, pulmonic stenosis,

OMIM (151100)

abnormal genitalia, short stature, sensorineural deafness.

General: CALM (50\%); mental retardations (mild); heart defects; thoracic deformities; small penis;

Sarkozy, 2008

hypoplastic ovarian; renal agenesis; spina bifida, delayed puberty.

$\boldsymbol{H} \boldsymbol{\&}$ : prognathism, triangular face, short neck; low-set ears; ptosis, epicanthal folds, strabismus; broad

nose; cleft palate.

Leopard syndrome 2

Typical: short stature, hypertrophic cardiomyopathy, craniofacial anomalies, lentigines, CALM.

General: cardiac changes; delayed puberty.

$\boldsymbol{H} \boldsymbol{\&} \boldsymbol{N}$ : dolichocephaly, prominent chin, short neck; low-set ears; downslanting palpebral fissures, hypertelorism.

Typical: pigmented lesions, hyperkeratosis, short stature, craniofacial anomalies.

General: lentigines, CALM, multiple nevi; cognitive deficits (mild); heart and thoracic defects; delayed

bone age.

H\&N: low-set ears, hearing loss; hypertelorism; depressed nasal bridge; short/webbed neck; curly hair.

Typical: combines features: neurofibromatosis and Noonan syndrome.

Neurofibromatosis-

Noonan syndrome

General: CALM, freckling, neurofibroma; developmental delay; short stature; pulmonic stenosis;

sternum defects; optic glioma.

$\boldsymbol{H} \boldsymbol{\&} \boldsymbol{N}$ : macrocephaly; face hypoplasia; low-seater ears; hypertelorism, downslanting palpebral fissures, ptosis, epicanthal folds, Lisch nodules; short neck.

Watson syndrome

Typical: pulmonary valvular stenosis, CALM, cognitive deficit, short stature.

General: multiple CALMs, neurofibromas, freckling.

$\boldsymbol{H} \boldsymbol{\&} \boldsymbol{N}$ : relative macrocephaly, Lisch nodules.

OMIM (611554)

OMIM (613707)

$\operatorname{OMIM}(601321)$

Nystron, 2009

Brems, 2007

OMIM(193520)

Hamm, 2019

Allanson, 1991 Highlighted changes: skin
and short stature

Others features

Ref.

Bloom syndrome

Typical: short stature; hypo/hyperpigmented skin, facial telangiectatic; predisposition to malignancy.

OMIM

(210900)

General: CALM, hypertrich
azoospermia; digital defects.

$\boldsymbol{H} \boldsymbol{\&} \boldsymbol{N}$ : dolichocephaly, microcephaly; narrow face; prominent ears and nose; absent upper lateral

incisors; high-pitched voice.

Rosales-Solis,

2016

Arora, 2014

Russell-Silver syndrome,

Typical: pigmentary anomaly, X-linked - severe in males, mild in females.

OMIM (312780)

$\mathrm{X}$ - linked

General: CALM; achromatic areas of trunk and limbs; prenatal growth retardation.

$\boldsymbol{H} \boldsymbol{\&} \boldsymbol{N}$ : triangular facies.

Turner syndrome

Typical: retarded growth, gonadal dysgenesis, infertility.

ORPHA (881)

General: CALM (association with NF1); ovarian failure; bone anomalies; lymphedema; cardiac/renal

Hatipoglu, 2010

anomalies; thyroid and gastrointestinal involvement.

$H \& N$ : round face, micrognathia, webbed neck, low posterior hairline; deafness.

Highlighted changes: skin,

Others features

Ref.

short stature, neurologic

Nijmegen $\quad$ breakage
syndrome

Typical: microcephaly, short stature, immunodeficiency, predisposition to cancer (lymphoma, glioma,

OMIM (251260)

syndrome

General: CALM, progressive vitiligo; mental retardation, hyperactivity, neurodegeneration; anal stenosis/atresia; primary ovarian failure.

$\boldsymbol{H} \boldsymbol{\&} \boldsymbol{N}$ : microcephaly, prominent midface, upward slanting of palpebral fissures; large dysplastic ears; choanal atresia, cleft lip/palate.

Nijmegen breakage syndrome-Like disorder

Johnson neuroectodermal syndrome

Noonan Syndrome-like disorder with loose anagen hair 2

Noonan Syndrome-like disorder with or without juvenile myelomonocytic leukemia
Typical: severe prenatal growth retardation and persistent postnatal growth restriction, congenital microcephaly, borderline to mildly impaired intellectual development, normal sexual development and radioresistant DNA synthesis with no immunodeficiency, myelodysplasia, or early neurodegeneration.

General: CALM, multiple pigmented nevi; short stature; developmental delay, spasticity, ataxia, Chiari malformation; brachydactyly, clinodactyly, sandal gap; widely spaced nipples; cutaneous vascular anomalies, Wolff-Parkinson-White anomaly.

$\boldsymbol{H} \boldsymbol{\&}$ : microcephaly, sloping forehead, micrognathia; hypertelorism; broad nasal bridge; hypoplastic nasal septum.

Typical: anosmia and hypogonadotropic hypogonadism, conductive deafness, alopecia, other variable anomalies.

General: hypohidrosis, multiple truncal CALM (rare); mental retardation; short stature; heart defect; hypogonadism; small penis.

$\boldsymbol{H} \boldsymbol{\&}$ : alopecia, microcephaly; protruding ear, microtia, external auditory canal atresia; absent eyebrows/eyelashes; choanal stenosis; cleft palate.

Typical: distinctive hair anomaly; heart defects; distinctive skin features; short stature.

General: freckling, CALM, hypopigmentation; loose/thin skin; developmental delay, Chiari I, DandyWalker malformation; delayed bone age.

$\boldsymbol{H} \boldsymbol{\&}$ : macrocephaly, craniosynostosis; preauricular pits, low-set ears; hypertelorism, downslanting palpebral fissures, ptosis; arched palate; short neck; sparse hair.

Typical: facial dysmorphism, wide spectrum of cardiac disease, reduced growth, variable cognitive deficits, ectodermal and musculoskeletal anomalies.

General: CALM, lymphedema; thin skin; hypotonia; delayed psychomotor development (mild); language delay; increased susceptibility to juvenile myelomonocytic leukemia; joint laxity, cubitus valgus; cryptorchidism; pectus excavatum, widely spaced nipples; congenital heart defects, aortic stenosis, mitral insufficiency.
OMIM(147770)

Hamm, 2019

OMIM (617506)

OMIM (613563) 
Microcephalic osteodysplastic primordial dwarfism type

Ataxia-telangiectasia

Noonan syndrome 6

Noonan syndrome 13
$\boldsymbol{H} \boldsymbol{\&} \boldsymbol{N}$ : thin hair; frontal bossing, triangular face, long philtrum; large ears, low-set ears; hypertelorism ptosis, downslanting palpebral fissures; depressed nasal bridge, thick lips, short neck, webbed neck.

Typical: severe short stature, microcephaly.

General: CALM, hypopigmentation; mental retardation, multiple aneurysms; narrow chest; digital defects, delayed bone age; short bones; premature puberty.

$H \& N$ : microcephaly; retrognathia; small ears; upward-slanting palpebral fissures; prominent nasal root; enamel hypoplasia, microdontia.

Typical: cerebellar cortical degeneration and ataxia, telangiectasias, immune defects, predisposition to cancer. Short stature.

General: CALM, sclerodermatous changes; dysarthric, choreoathetosis, myoclonus, seizures; lymphoma, leukemia; immunodeficiency; hypogonadism.

$\boldsymbol{H} \boldsymbol{\&}$ : ocular telangiectasia, oculomotor abnormalities; progeric changes.

Typical: short stature, facial dysmorphism, heart defects.

General: keratosis pilaris, hyperkeratosis, lentigines, CALM; developmental delay; cardiac defects; thorax deformity; juvenile myelomonocytic leukemia, polyhydramnios and single umbilical artery.

$\boldsymbol{H} \boldsymbol{\&} \boldsymbol{N}$ : macrocephaly, low-set ears, hearing loss; epicanthal folds, downslanting palpebral fissures, long eyebrows; broad nasal bridge; webbed neck; sparce hair.

Typical: developmental delay and impaired intellectual development, associated with behavioral problems. Reduced postnatal growth and craniofacial anomalies.

General: multiple lentigines, CALM, hypochromic spots; hypotonia, seizures, attentiondeficit/hyperactivity disorder, autism spectrum disorder, anxiety; cubitus valgus, pes planus hypospadias, cryptorchidism; broad thorax, scapular winging, widely spaced nipples; heart defects prolapse; hypertrichosis; low posterior hairline.

$\boldsymbol{H} \boldsymbol{\&} \boldsymbol{N}$ : high/broad forehead, long philtrum; low-set ears; ptosis, hypertelorism, epicanthal folds; wide nasal bridge, everted lower lip; dental changes; short neck.
Highlighted changes: skin
short stature, neurologic

Johanson-Blizzard

syndrome

Cardiofaciocutaneous syndrome 1

Costello syndrome

Mismatch repair cancer syndrome (MMRCS1, MMRCS2, MMRCS3 MMRCS4)

Tuberous sclerosis 1
Typical: short stature, mental retardation, variable dysmorphic features.

General: CALM, scalp aplasia cutis, transverse palmar crease; hypotonia; heart defect; small nipples, absent areolae; liver failure; pancreatic insufficiency; imperforate anus; micropenis; delayed bone age, clinodactyly.

$H \& N$ : microcephaly; hearing loss; strabismus, cutaneous-lacrimal fistulae; beaked nose; hypoplastic teeth, absent permanent; blonde/unruly hair.

Typical: tetraphocomelia, growth retardation, mental retardation, craniofacial/cardiac/renal anomalies General: midfacial capillary hemangioma, CALM; encephalocele, hydrocephalus; heart defect; rudimentary gallbladder; enlarged penis or clitoris; polycystic kidney; digital defects, talipes equinevalgus; nuchal cystic hygroma.

$\boldsymbol{H} \& \boldsymbol{N}$ : microcephaly, brachycephaly, craniosynostosis; malformed ears; hypertelorism, bluish sclerae widened nasal bridge; cataract, downslanting palpebral fissures; cleft lip/palate, short neck; silvery blonde sparse hair.

Typical: distinctive coarse facies, heart defects, mental retardation. Ectodermal abnormalities. Short stature.

General: atopic dermatitis, ichthyosis, hyperkeratosis, cavernous hemangioma, multiple palmar creases, lentigines, CALM (9-31\%); seizures, hypotonia/hypertonia, cortical atrophy, hypoplasia corpus callosum; peripheral axonal neuropathy; delayed bone age, clinodactyly.

$H \& N$ : macro/dolichocephaly; micrognathia; low-set ears, hearing loss; ptosis, nystagmus, strabismus, downslanting palpebral fissures, hypertelorism, epicanthal folds, loss of visual acuity; absence eyebrows/eyelashes; cleft palate, crossbite; sparse hair

Typical: characteristic coarse facies, short stature, distinctive hand posture and appearance, severe feeding difficulty. Significant cancer risk.

General: cutis laxa, redundant skin, deep palmar/plantar creases, CALM (rare), papilloma, acanthosis nigricans, palmar nevi; mental retardation, cerebral atrophy, cerebellar tonsillar herniation; cardiac defect; small lung; renal failure; wide distal phalanges; nail changes.

$\boldsymbol{H} \& \boldsymbol{N}$ : macrocephaly, micrognathia; low-set ears; hypertelorism, epicanthal folds, downslanting palpebral fissures, ptosis, strabismus; macroglossia; depressed nasal neck; sparce hair; hoarse voice.

Typical: cancer predisposition syndrome with 4 main tumor types: hematologic malignancies, brain tumors, colorectal tumors, multiple intestinal polyps.

General: CALM, freckling, neurofibromas, plexiform neurofibromas; agenesis of the corpus callosum, gray matter heterotopia, intracerebral cysts; colonic polyps; ependymoma, glioblastoma, oligodendroglioma, neuroblastoma, astrocytoma, medulloblastoma, basal cell carcinoma, colonic adenocarcinoma, leukemia, lymphoma.

$H \& N$ : no specific changes.

Typical: hamartomas in multiple organ systems

General: white ash leaf-shaped macules, subcutaneous nodules, CALM, subungual fibromata; hamartomatous (brain), subependymal nodules, cortical tubers, attention- deficit hyperactivity disorder; seizures, mental retardation, intracranial calcification, autism; Wolf-Parkinson-White; kidney tumors, myocardial rhabdomyoma, renal carcinoma, ependymoma, astrocytoma, benign tumors; precocious puberty.
$\operatorname{OMIM}(210720)$

Nishimura,

2003

Young, 2004

OMIM (208900)

OMIM (613224)

OMIM (619087)

Ref.

OMIM (243800)

OMIM (268300)

OMIM (115150)

Hamm, 2019

Zhang, 2016

OMIM(218040)

Zhang, 2016

OMIM (276300 619096,619097, 619101)

Hamm, 2019

OMIM (191100) 
Tuberous sclerosis 2

Neurofibromatosis, type III

Waardenburg syndrome type $2 \mathrm{E}$

Chromosome 17q11.2 deletion syndrome

Mulchandani-BhojConlin syndrome

$H \boldsymbol{N}$ : facial angiofibroma, retinal astrocytoma, optic gliomas; gingival fibroma.

Highlighted changes: skin short stature, neurologic

Silver-Russell syndrome

Chromosome 15q26qter deletion syndrome

Seckel syndrome 2

Rubinstein-Taybi syndrome

Kabuki syndrome 1

Microcephaly, growth restriction and increased sister chromatid exchange 2
Typical: hamartomas in multiple organ systems. More severe disease than type-1.

General: white ash leaf-shaped macules, subcutaneous nodules, CALM, subungual fibromata; hamartomatous (brain), subependymal nodules, cortical tubers, seizures, mental retardation, intracranial calcification, attention-deficit hyperactivity disorder, autism; Wolf-Parkinson-White; kidney tumors, myocardial rhabdomyoma, renal carcinoma, ependymoma, astrocytoma, benign tumors; precocious puberty.

$H \& N$ : facial angiofibroma, retinal astrocytoma, optic gliomas, gingival fibroma.

Typical: bilateral acoustic neuromas, posterior fossa and upper cervical meningiomas, and spinal/paraspinal neurofibromas, but optic gliomas have not been seen.

General: large CALM, freckling, palmar neurofibromas: spinal/paraspinal neurofibromas, cervical meningiomas.

$\boldsymbol{H} \boldsymbol{\&} \boldsymbol{N}$ : bilateral acoustic neuromas. No íris Lisch nodules.

Typical: auditory-pigmentary syndrome characterized by pigmentary abnormalities, congenital hearing loss $(100 \%)$, neurologic abnormalities

General: hypopigmented patches, CALM, freckles, premature graying; mental retardation, axial hypotonia, brain hypomyelination, ataxia.

$\boldsymbol{H} \boldsymbol{\&} \boldsymbol{N}$ : temporal bone abnormalities; hypopigmented iris/retina, bright blue eyes, nystagmus; white forelock/eyelashes/eyebrows; large incisors, irregular dentition.

Typical: variable facial dysmorphism, mental retardation, excessive number neurofibromas, increased risk for malignant peripheral nerve tumors.

General: CALM (93\%), freckling; attention-deficit hyperactivity disorder; tall stature; heart defects; pectus excavatum; bone cysts, large hands/feet; hypotonia.

H\&N: macrocephaly; coarse facies; Lisch nodules (93\%), hypertelorism, optic glioma.

Typical: short stature, feeding difficulties.

General: CALM (infrequent); hypotonia; horseshoe kidney; digital defects.

$\boldsymbol{H} \boldsymbol{\&} \boldsymbol{N}$ : microcephaly; mild facial dysmorphism: epicanthal folds, dolichocephaly with retrognathia.

OMIM (613254)

OMIM (162260)

OMIM (611584)

OMIM (613675)

\section{OMIM (617352)}

Mulchandani,2016

Ref.

Typical: severe growth retardation, craniofacial features, body asymmetry, others minor malformations.

General: CALM; developmental delay; cardiac defects; digital defects; craniopharyngioma, testicular seminoma, hepatocellular carcinoma, Wilms tumor.

$\boldsymbol{H} \boldsymbol{\&}$ : craniofacial disproportion, pseudo-hydrocephalic appearance; blue sclera.

Typical: deletion of chromosome 15q26-qter encompassing the insulin-like growth factor 1 receptor gene. Short stature is stablished hallmark.

General: CALM; mental retardation; congenital cardiac anomalies; micropenis, hypospadias, cryptorchidism; digital defects.

$H \boldsymbol{H}$ : microcephaly; low-set ears; blepharophimosis, strabismus; broad bridge nose.

Typical: short stature, growth retardation, microcephaly, mental retardation, characteristic facial appearance.

General: CALM; cerebellar hypoplasia, calcifications; ectopic kidneys; digital defects.

$\boldsymbol{H} \boldsymbol{\&} \boldsymbol{N}$ : microcephaly, short anterior cranial base; prominent nose; macroglossia; small teeth; highpitched voice.

Typical: mental retardation, postnatal growth deficiency, microcephaly, broad thumbs /halluces, dysmorphic facial features.

General: single transverse palmar creases, CALM; seizures; heart defect; sternal anomalies; spina bifida, small iliac wings, digital defects; hirsutism.

$\boldsymbol{H} \boldsymbol{\&} \boldsymbol{N}$ : microcephaly, frontal bossing, low anterior hairline; low-set ears, hearing loss; arched eyebrows, long eyelashes, ptosis, epicanthal folds, strabismus, cataracts, downslanting palpebral fissures; beaked nose; dental crowding, talon cusps, screwdriver incisors, enamel hypoplasia.

Typical: mental retardation syndrome with additional features postnatal dwarfism, peculiar facies, characteristic skeletal changes. Hearing loss.

General: CALM, cutis aplasia; seizures, hypotonia; short stature; heart defect; anal defects; small penis, renal anomalies; vertebral/hip anomalies, digital defects; hypothyroidism, premature thelarche; hirsutism.

$\boldsymbol{H} \boldsymbol{\&} \boldsymbol{N}$ : microcephaly; large ears, preauricular pit; long palpebral fissures, eversion of eyelids, thick eyelashes, ptosis, blue sclerae, broad eyebrows; cleft.

Typical: growth restriction with short stature, microcephaly.

OMIM (618097)

OMIM (147920)

Ghada, 2011

General: CALM; mild developmental delayed; dilated cardiomyopathy.

$\boldsymbol{H} \& \boldsymbol{N}$ : microcephaly; dysmorphic facial features progeroid-like.
OMIM (180860)

Spiteri, 2017

OMIM (612626)

Veenma, 2010

OMIM (606744)

Veenma, 2010

OMIM (180849) 
OHDO syndrome, $\mathrm{X}$ linked

Autosomal recessive primary microcephaly

Smith-Kingsmore syndrome

Developmental delay, intellectual disability, obesity, and dysmorphism

Ring chromosome 14 syndrome

Genetic susceptibility to neuroblastoma

\begin{abstract}
Typical: distinct blepharophimosis-mental retardation syndrome.
General: CALM; developmental delay; low weight; scrotal hypoplasia; clinodactyly.

$\boldsymbol{H} \boldsymbol{\&} \boldsymbol{N}$ : coarse facial features; deafness; ptosis; wide nasal bridge, large nose; microstomia, micrognathia.
\end{abstract}

Typical: microcephaly, developmental delay, variable dysmorphic facies.

General: CALM; behavioral problems; small cerebral cortex, simplified gyral pattern, partial absence of the corpus callosum, tonic clonic seizures.

$H \& N$ : microcephaly; conical-shaped and widely spaced teeth; hearing loss (some); prominent nose.

Typical: macrocephaly, seizures, umbilical hernia, facial dysmorphic features.

General: CALM; intellectual disability, hypogenesis of the corpus callosum, polymicrogyria, heterotopic gray matter, autistic features; small thorax; diastasis recti, umbilical hernia; limb shortening, deep palmar/plantar creases, short phalanges, small toenails; hypotonia.

$\boldsymbol{H} \boldsymbol{\&} \boldsymbol{N}$ : macrocephaly, frontal bossing; midface hypoplasia; hypertelorism, downslanting palpebral fissures, strabismus; short nose; curly hair.

Typical: developmental delay, intellectual disability, behavioral abnormalities, dysmorphic features, obesity.

General: CALM; attention deficit-hyperactivity disorder; hands: tapering fingers, clinodactyly; feet: syndactyly; hypotonia.

$\boldsymbol{H} \boldsymbol{\&} \boldsymbol{N}$ : micrognathia; large ears; thick eyebrows, synophrys, hypertelorism, upslanting palpebral fissures, epicanthal folds, strabismus, nystagmus.

Typical: early-onset epilepsy, developmental delay with mental retardation, microcephaly, dysmorphic facial features.

General: Pigmentary abnormalities (in some), CALM; short stature (less frequently observed signs); hypotonia, seizures, intellectual disability, poor speech.

$\boldsymbol{H} \boldsymbol{\&} \boldsymbol{N}$ : microcephaly,dolichocephaly; low-set ears; downslanting palpebral fissures, epicanthal folds, hypertelorism; flat nasal bridge, anteverted nostrils. Short neck.

Typical: genetic predisposition to neuroblastoma tumors.

General: bluish skin nodules, CALM; paraneoplastic syndromes, opsoclonus, myoclonus, ataxia, spinal cord compression; neuroblastoma; ganglioneuroma.

$\boldsymbol{H} \& \boldsymbol{N}$ : Horner's syndrome.
OMIM (300895)

OMIM (604804)

Pagnamenta, 2012

OMIM (616638)

OMIM (617991)

Jansen, 2018

OMIM (616606)

Rinaldi, 2017

Shah, 2010

OMIM (256700)

Origone, 2003

Chatten, 1967

Highlighted changes:
endocrine

Pheochromocytoma-islet

cell tumor syndrome

Typical: : autosomal dominant endocrine adenomatosis.

Others features

Ref.

General: CALM, freckling; cerebral hemorrhage; tachycardia, congestive heart failure, hypertension pheochromocytoma, islet cell tumor.

$\boldsymbol{H} \boldsymbol{\&} \boldsymbol{N}$ : hypertensive retinopathy.

Pheochromocytoma

Typical: catecholamine-secreting tumors that usually arise within the adrenal medulla.

OMIM (171300)

General: sweating, CALM, hemangiomata; cerebral hemorrhage; renal artery stenosis, tachycardia, congestive heart failure; hypertension.

$\boldsymbol{H} \boldsymbol{\&} \boldsymbol{N}$ : hypertensive retinopathy, retinal angiomatosis, cataract.

Adiposis dolorosa

Typical: generalized obesity.

General: lipomas, CALM; chronic pain, fatigue, sleep disturbances; depression, anxiety; arthralgia; muscle aches.

$H \& N$ : no specific changes.

Multiple endocrine neoplasia type I

Multiple endocrine neoplasia type IIB

Typical: tumors: parathyroid, pancreatic, duodenal endocrine cells; anterior pituitary adenomas.

General: lipomas, collagenomas, CALM, hypopigmented macules; adenoma: parathyroid, adrenocortical; prolactinoma, gastrinoma, carcinoid tumors.

$\boldsymbol{H} \boldsymbol{\&}$ : facial angiofibromas, multiple gingival papules.

Typical: hamartoneoplastic syndrome characterized by aggressive medullary thyroid carcinoma, pheochromocytoma, mucosal neuromas, thickened corneal nerves.

General: CALM; ganglioneuroma, myopathy, developmental delay; failure to thrive; parathyroid hyperplasia; goiter; colonic diverticulum.

$\boldsymbol{H} \boldsymbol{\&}$ : coarse-appearing facies; pedunculated nodules (eyelid), eyelid/corneal neuromas; anteverted eyelid; large eyebrow; neuromas of lips/tongue.

\begin{tabular}{ll}
\hline \multicolumn{1}{c}{ Miscellaneous } & \multicolumn{1}{c}{ Others features } \\
\hline Fanconi anemia & Typical: developmental abnormalities in major organ systems, early-onset bone marrow failure, high \\
(FANCA, FANCC, & predisposition to cancer (leukemia). \\
FANCD2, FANCI) & skin (hyperpigmentation, CALM), urogenital, cardiopulmonary, gastrointestinal, central nervous \\
& systems. \\
& H\&N: microcephaly; strabismus, microphthalmia; short neck; "Fanconi facies"; ear malformations. \\
& Typical: severe anemia requiring regular transfusions. Erythropoietic hyperplasia of bone marrow. \\
Anemia, hypochromic & General: CALM; growth retardation; dysfunction of hypothalamo-pituitary-gonadal axis; hematologic \\
microcytic, with iron & alterations. \\
overload 2 & H\&N: no specific changes.
\end{tabular}

H\&N: Head \& Neck; CALM(s): café-au-lait macule(s); OMIM: Online Mendelian Inheritance in Man; ORPHA: rare disease nomenclature. Source: Authors. 
Neurological disorders were observed in 32 types of diseases that present CALM, which may include a wide spectrum of changes, such as cognitive impairment, mental retardation, developmental delay, multiple aneurysms, cerebellar cortical degeneration, cerebellar ataxia, seizures, central nervous system malformations, brain tumors, hyperactivity, autistic spectrum disorders, peripheral nerve tumors, and other disturbances. Short stature was a characteristic observed in 28 of the identified genetic syndromes. Other relevant clinical findings observed in CALM-associated syndromes included predisposition to cancer (observed in 16 of 60 types of syndromes) and endocrine disturbances. Changes in the head and neck assessment were found in 52 syndromes linked to the presence of CALM.

The OMIM number, mode of inheritance, chromosome, mutated genes, and affected proteins for the 60 identified diseases that may exhibit CALM in their clinical presentation are listed in Table 2 (grouped according to mutated gene classification). The most common mode of inheritance for syndromes with CALM is autosomal dominant, occurring in $68.3 \%$ (41 of 60) of these genetic disorders. An autosomal recessive mode of inheritance was detected in $21.6 \%$ (13 of 60 ) of the syndromes, with the remainder being X-linked cases, isolated cases, or unestablished patterns of inheritance.

Table 2. Genetic syndromes reported with cafe-au-lait spots: genetics

\begin{tabular}{|c|c|c|c|c|c|}
\hline Syndrome & OMIM\# & $M O I$ & Chromosome & Gene & Protein \\
\hline \multicolumn{6}{|l|}{ RAS/MAPK signal transduction pathway } \\
\hline Neurofibromatosis type I & $\# 162200$ & $\mathrm{AD}$ & $17 q 11.2$ & $N F 1$ & Neurofibromin \\
\hline Legius syndrome & \#611431 & $\mathrm{AD}$ & $15 q 14$ & SPRED1 & $\begin{array}{l}\text { Sprouty-related, EVH1 domain- } \\
\text { containing protein } 1\end{array}$ \\
\hline Leopard syndrome 1 & $\# 151100$ & $\mathrm{AD}$ & $12 q 24.13$ & PTPN11 & $\begin{array}{l}\text { Tyrosine-protein phosphatase non- } \\
\text { receptor type } 11\end{array}$ \\
\hline Leopard syndrome 2 & \#611554 & $\mathrm{AD}$ & $3 \mathrm{p} 25.2$ & $R A F 1$ & $\begin{array}{l}\text { RAF proto-oncogene serine/threonine- } \\
\text { protein kinase }\end{array}$ \\
\hline Leopard syndrome 3 & \#613707 & $\mathrm{AD}$ & $7 \mathrm{q} 34$ & $B R A F$ & Serine/threonine-protein kinase B-raf \\
\hline Neurofibromatosis-Noonan syndrome & \#601321 & $\mathrm{AD}$ & $17 q 11.2$ & $N F 1$ & Neurofibromin \\
\hline Watson syndrome & $\# 193520$ & $\mathrm{AD}$ & $17 q 11.2$ & $N F 1$ & Neurofibromin \\
\hline $\begin{array}{l}\text { Noonan syndrome-like disorder with loose anagen } \\
\text { hair } 2\end{array}$ & \#617506 & $\mathrm{AD}$ & $2 \mathrm{p} 23.2$ & $P P P 1 C B$ & $\begin{array}{l}\text { Serine/threonine-protein phosphatase } \\
\text { PP1-beta catalytic subunit }\end{array}$ \\
\hline Noonan syndrome 6 & \#613224 & $\mathrm{AD}$ & $1 \mathrm{p} 13.2$ & $N R A S$ & GTPase NRas \\
\hline Cardiofaciocutaneous syndrome 1 & \#115150 & $\mathrm{AD}$ & $7 \mathrm{q} 34$ & BRAF & Serine/threonine-protein kinase B-raf \\
\hline Costello syndrome & \#218040 & $\mathrm{AD}$ & $11 \mathrm{p} 15.5$ & $H R A S$ & GTPase HRas \\
\hline
\end{tabular}

\section{KIT receptor tyrosine kinase ligand signaling pathways}

\begin{tabular}{|c|c|c|c|c|c|}
\hline Familial progressive hyperpigmentation & $\# 614233$ & $\mathrm{AD}$ & 19p13-pter & KITLG & Kit ligand \\
\hline $\begin{array}{l}\text { Familial progressive hyperpigmentation } \\
\text { and hypopigmentation }\end{array}$ & $\# 145250$ & $\mathrm{AD}$ & $12 q 21.32$ & $K I T L G$ & Kit ligand \\
\hline \multirow{2}{*}{ Piebald trait (piebaldism) } & \#172800 & $\mathrm{AD}$ & $4 q 12$ & $K I T$ & Mast/stem cell growth factor receptor Kit \\
\hline & & $\mathrm{AD}$ & $8 \mathrm{q} 11.21$ & SNAI2 & Zinc finger protein SNA12 \\
\hline Waardenburg syndrome type $2 \mathrm{E}$ & \#611584 & $\mathrm{AD}$ & $22 q 13.1$ & SOX10 & Transcription factor SOX-10 \\
\hline
\end{tabular}

\section{PHAKOMATOSES}

Neurofibromatosis type II
McCune-Albright syndrome
Cowden syndrome 1
Peutz-Jeghers syndrome

$\begin{array}{lccl}\# 101000 & \text { AD } & 22 \mathrm{q} 12.2 & \text { NF2 } \\ \# 174800 & \text { Sporadic } & 20 \mathrm{q} 13.32 & \text { GNAS } \\ \# 158350 & \text { AD } & 10 \mathrm{q} 23.31 & \text { PTEN } \\ \# 175200 & \text { AD } & 19 \mathrm{p} 13.3 & \text { STK11 }\end{array}$




\begin{tabular}{|c|c|c|c|c|c|}
\hline Ataxia-telangiectasia & \#208900 & $\mathrm{AR}$ & $11 \mathrm{q} 22.3$ & $A T M$ & Serine-protein kinase ATM \\
\hline Tuberous sclerosis 1 & \#191100 & $\mathrm{AD}$ & $9 q 34.13$ & TSC1 & Hamartin \\
\hline \multirow[t]{2}{*}{ Tuberous sclerosis 2} & \#613254 & $\mathrm{AD}$ & $12 q 15$ & $I F N G$ & IFN- $\gamma$ \\
\hline & & & $16 \mathrm{p} 13.3$ & $T S C 2$ & Tuberin \\
\hline \multicolumn{6}{|l|}{ MISCELLANEOUS } \\
\hline Adams-Oliver syndrome 4 & \#615297 & $\mathrm{AR}$ & $3 \mathrm{p} 14.1$ & $E O G T$ & $\begin{array}{l}\text { EGF domain-specific O-linked N- } \\
\text { acetylglucosamine transferase }\end{array}$ \\
\hline Carney complex & \#160980 & $\mathrm{AD}$ & $17 \mathrm{q} 24.2$ & PRKAR1A & $\begin{array}{l}\text { cAMP-dependent protein kinase type I- } \\
\text { alpha regulatory subunit }\end{array}$ \\
\hline Syndrome & OMIM\# & $M O I$ & Chromosome & Gene & Protein \\
\hline \multicolumn{6}{|l|}{ MISCELLANEOUS } \\
\hline Bloom syndrome & \#210900 & $\mathrm{AR}$ & $15 q 26.1$ & $R E C Q L 3$ & Bloom syndrome protein \\
\hline Nijmegen breakage syndrome & \#251260 & $\mathrm{AR}$ & $8 \mathrm{q} 21.3$ & $N B N$ & Nibrin \\
\hline Nijmegen breakage syndrome-Like disorder & \#613078 & $\mathrm{AR}$ & $5 q 31.1$ & $R A D 50$ & DNA repair protein RAD50 \\
\hline $\begin{array}{l}\text { Microcephalic osteodysplastic primordial dwarfism } \\
\text { type II }\end{array}$ & \#210720 & AR & $21 \mathrm{q} 22.3$ & PCNT & Pericentrin \\
\hline Johanson-Blizzard Syndrome & \#243800 & $\mathrm{AR}$ & $15 q 15.2$ & $U B R 1$ & E3 ubiquitin-proteinligase UBR1 \\
\hline Noonan syndrome 13 & \#619087 & $\mathrm{AD}$ & $22 q 11.22$ & $M A P K 1$ & Not reported \\
\hline Noonan Syndrome-like disorder with or & \#613563 & $\mathrm{AD}$ & $11 \mathrm{q} 23.3$ & $C B L$ & E3 ubiquin-protein ligase CBL \\
\hline \multicolumn{6}{|l|}{ without juvenile myelomonocytic leukemia } \\
\hline Seckel syndrome 2 & \#606744 & $\mathrm{AR}$ & $18 \mathrm{q} 11.2$ & $R B B P 8$ & DNA endonuclease RBBP8 \\
\hline Roberts syndrome & $\# 268300$ & AR & $8 \mathrm{p} 21.1$ & ESCO2 & $\mathrm{N}$-acetyltransferase ESCO2 \\
\hline \multirow[t]{4}{*}{ Mismatch repair cancer syndrome } & \#276300 & $\mathrm{AR}$ & $3 \mathrm{p} 22.2$ & $M L H 1$ & Proteína Mlh1 \\
\hline & & & $2 \mathrm{p} 21-\mathrm{p} 16$ & $M S H 2$ & Protein Msh2 \\
\hline & & & $2 \mathrm{p} 16$ & MSH6 & Protein Msh6 \\
\hline & & & $7 \mathrm{p} 22$ & $P M S 2$ & Endonuclease PMS2 \\
\hline
\end{tabular}

\begin{tabular}{|c|c|c|c|c|c|}
\hline MISCELLANEOUS & & & & & \\
\hline Chromosome 15q26-qter deletion syndrome & $\# 612626$ & IC & $15 \mathrm{q} 26$-qter & IGF1R & Not reported \\
\hline Chromosome 17q11.2 deletion syndrome & $\# 613675$ & $\mathrm{AD}$ & $17 \mathrm{q} 11.2$ & Not reported & Not reported \\
\hline Rubinstein-Taybi syndrome 1 & $\# 180849$ & $\mathrm{AD}$ & $16 \mathrm{p} 13.3$ & CREBBP & CREB-binding protein \\
\hline Kabuki syndrome 1 & \#147920 & $\mathrm{AD}$ & $12 \mathrm{q} 13.12$ & $K M T 2 D$ & $\begin{array}{l}\text { Histone-lysine N-methyltransferase } \\
\text { 2D }\end{array}$ \\
\hline $\begin{array}{l}\text { Microcephaly, growth restriction, and } \\
\text { increased sister chromatid } \\
\text { exchange } 2\end{array}$ & \#618097 & AR & $17 \mathrm{p} 11.2$ & TOP3A & DNA topoisomerase 3-alpha \\
\hline Ohdo syndrome, $\mathrm{X}$-linked & \#300895 & XLR & $\mathrm{Xq} 13.1$ & MED12 & $\begin{array}{l}\text { Mediator of RNA polymerase II } \\
\text { transcription subunit } 12\end{array}$ \\
\hline Autosomal recessive primary microcephaly & \#604804 & $\mathrm{AR}$ & $9 \mathrm{q} 33.2$ & CDK5RAP2 & $\begin{array}{l}\text { CDK5 regulatory subunit-associated } \\
\text { protein } 2\end{array}$ \\
\hline Smith-Kingsmore syndrome & $\# 616638$ & $\mathrm{AD}$ & $1 \mathrm{p} 36.22$ & MTOR & $\begin{array}{l}\text { Serine/threonine-protein kinase } \\
\text { mTOR }\end{array}$ \\
\hline $\begin{array}{l}\text { Developmental delay, intellectual disability, } \\
\text { obesity, and dysmorphism }\end{array}$ & \#617991 & $\mathrm{AD}$ & $6 \mathrm{q} 14.1$ & PHIP & PH-interacting protein \\
\hline Ring chromosome 14 syndrome & \#616606 & IC & Chr.14 & $R C 14 R$ & Not reported \\
\hline & & & $14 \mathrm{q} 23.3$ & $M A X$ & Protein max \\
\hline Genetic susceptibility to neuroblastoma & $\# 256700$ & $\mathrm{AD}$ & $1 \mathrm{p} 36.22$ & $K I F 1 B$ & Kinesin-like protein KIF1B \\
\hline Multiple endocrine neoplasia type 1 & $\# 131100$ & $\mathrm{AD}$ & $11 \mathrm{q} 13.1$ & MEN1 & Menin \\
\hline
\end{tabular}




\begin{tabular}{|c|c|c|c|c|c|}
\hline Multiple endocrine neoplasia type 2B & \#162300 & $\mathrm{AD}$ & $10 q 11.21$ & RET & $\begin{array}{l}\text { Proto-oncogene tyrosine-protein } \\
\text { kinase receptor Ret }\end{array}$ \\
\hline Syndrome & OMIM\# & MOI & Chromosome & Gene & Protein \\
\hline \multicolumn{6}{|l|}{ MISCELLANEOUS } \\
\hline \multirow[t]{8}{*}{ Pheochromocytoma } & $\# 171300$ & $\mathrm{AD}$ & $1 \mathrm{p} 36.22$ & $K I F 1 B$ & Kinesin-like protein KIF1B \\
\hline & & & $1 \mathrm{p} 36.13$ & $S D H B$ & $\begin{array}{l}\text { Succinate dehydrogenase iron-sulfur } \\
\text { subunit, mitochondrial }\end{array}$ \\
\hline & & & $2 \mathrm{q} 11.2$ & TMEM127 & Transmembrane protein 127 \\
\hline & & & $3 \mathrm{p} 25.3$ & $V H L$ & $\begin{array}{l}\text { Von Hippel-Lindau disease tumor } \\
\text { suppressor }\end{array}$ \\
\hline & & & $5 \mathrm{p} 13.2$ & $G D N F$ & $\begin{array}{l}\text { Glial cell line-derived neurotrophic } \\
\text { factor }\end{array}$ \\
\hline & & & $10 q 11.21$ & $R E T$ & $\begin{array}{l}\text { Proto-oncogene } \\
\text { kinase receptor Ret }\end{array}$ \\
\hline & & & $11 \mathrm{q} 23.1$ & $S D H D$ & $\begin{array}{l}\text { Succinate dehydrogenase cytochrome } \\
\text { b subunit mitochondrial }\end{array}$ \\
\hline & & & $14 \mathrm{q} .23 .3$ & $M A X$ & Protein max \\
\hline Silver-Russell syndrome 1 & \#180860 & $\mathrm{AD}$ & $11 \mathrm{p} 15.5$ & ICRI & Insulin-like growth factor II \\
\hline Mulchandani-Bhoj-Conlin syndrome & \#617352 & $\mathrm{AD}$ & $20 q 11-q 13$ & Not reported & Not reported \\
\hline Fanconi anemia (FA), complementation group A & \#227650 & AR & $16 \mathrm{q} 24.3$ & FANCA & FA group A protein \\
\hline FA, group C & \#227645 & AR & $9 q 22.32$ & FANCC & FA group $\mathrm{C}$ protein \\
\hline FA, group I & \#609053 & $\mathrm{AR}$ & $15 \mathrm{q} 26.1$ & FANCI & FA group I protein \\
\hline FA, group D2 & \#227646 & AR & $3 \mathrm{p} 25.3$ & FANCD2 & FA group D2 protein \\
\hline $\begin{array}{l}\text { Anemia, hypochromic microcytic, with iron } \\
\text { overload } 2\end{array}$ & \#615234 & $\mathrm{AD}$ & $2 q 14.2$ & STEAP3 & Metalloreductase STEAP3 \\
\hline \multicolumn{6}{|l|}{ UNKNOWN GENES } \\
\hline Multiple cafe-au-lait spots & $\# 114030$ & $\mathrm{AD}$ & Unknown & Unknown & Unknown \\
\hline Gastrocutaneous syndrome & \#137270 & $\mathrm{AD}$ & Unknown & Unknown & Unknown \\
\hline Neurofibromatosis type III & \#162260 & $\mathrm{AD}$ & Unknown & Unknown & Unknown \\
\hline Russell-Silver syndrome, X-linked & \#312780 & X-linked & Chromosome X & Unknown & Unknown \\
\hline Turner syndrome & ORPHA 881 & X-linked & Chromosome X & Unknown & Unknown \\
\hline Johnson neuroectodermal syndrome & \#147770 & $\mathrm{AD}$ & Unknown & Unknown & Unknown \\
\hline Pheochromocytoma-islet cell tumor syndrome & \#171420 & $\mathrm{AD}$ & Unknown & Unknown & Unknown \\
\hline Adiposis dolorosa & \#103200 & AD/sporadically & Unknown & Unknown & Unknown \\
\hline
\end{tabular}

Source: Authors.

\section{Discussion}

Genetic disorders account for approximately $80 \%$ of all rare diseases (Giugliani et al., 2019). All syndromes associated with the presence of CALM listed here are rare. There is no single definition for rare diseases. Within health systems, rare diseases have been defined based on the criteria of prevalence or number of affected individuals. According to the European Union, rare diseases are defined as those that affect less than 1 in 2,000 people (Giugliani et al., 2019). For the World Health Organization, a rare disease is one that affects up to 65 people in 100,000 individuals or 1.3 people in every 2,000 individuals. In the United States, legislation defines rare diseases strictly according to prevalence, specifically as "any disease or condition that affects fewer than 200,000 people in the United States". Brazil follows the same definition of rare diseases adopted by the World Health Organization (Alawi, 2019; Martelli, 2019).

Among all identified syndromes, neurofibromatosis type 1 is the most common, with a worldwide incidence ranging from 1 in 2,500 to 1 in 3,000 individuals (OMIM). It is the disease in which the association with CALM is well-recognized and considered a diagnostic hallmark (Shah, 2010; Zhang et al., 2016). However, in the presence of early-onset and multiple CALMs, several other genetic syndromes should be excluded (but not limited to), such as neurofibromatosis type II (Ferner, 2007; Evans, 2009; Madson, 2012; Plana-Pla, Bielsa-Marsol \& Carrato-Monino, 2017; Hamm et al., 2019), neurofibromatosis type III (Madson, 2012), Legius syndrome (neurofibromatosis type 1-like syndrome) (Madson, 2012; Zhang et al., 2016), 
multiple cafe-au-lait spots (Orphanet; Madson, 2012), McCune-Albright syndrome (Shah, 2010; Madson, 2012; Hamm et al., 2019), Noonan syndrome (Madson, 2012), Leopard syndrome (Madson, 2012; Zhang et al., 2016; Hamm et al., 2019), gastrocutaneous syndrome (Hamm et al., 2019), familial progressive hyperpigmentation and hypopigmentation (Amyere et al., 2011), familial progressive hyperpigmentation (Zhang et al., 2016), mismatch repair cancer syndrome (Shah, 2010; Baas et al., 2013; Hamm et al., 2019), Watson syndrome (Madson, 2012; Hamm et al., 2019) and cardiofaciocutaneus syndrome 1 (Hamm et al., 2019).

Most of these diseases that present multiple CALMs are part of the developmental diseases known as RASopathies. These diseases, which are associated with germline genetic modifications, comprise a group of clinically and genetically related diseases that present mutations and deletions associated with protein-coding genes that lead to activation and/or dysregulation of the Ras/mitogen-activated protein kinase (RAS/MAPK) pathway (Tajan, Paccoud, Branks, Edouard, \& Yart, 2018). Neurofibromatosis type I is caused by loss-of-function mutations of the NF1 gene that encodes neurofibromin (Picardo \& Cardinali, 2011); Legius syndrome results from inactivating mutations in the SPRED1 gene (Zhang et al., 2016; Tajan et al., 2018); Noonan syndrome caused by mutations in PTPN11, SOS1, RAF1, KRAS, NRAS, BRAF, RIT1, and RRAS genes (Zhang et al., 2016; Cao, Alrejaye, Klein, Goodwin \& Oberol, 2017; Tajan et al., 2018); Leopard syndrome, due to mutations in PTPN11, $R A F 1$, and $B R A F$ genes (Hamm et al., 2019); Costello syndrome due to activating mutations in the HRAS gene (Aoki, Niihori, Inoue \& Matsubara, 2016; Zhang et al., 2016); cardiofaciocutaneous syndrome resulting from gain-of-function mutations in the $B R A F, K R A S$, and MAP2K1 or MAP2K2 genes (Digilo et al., 2011; Aoki et al., 2016; Zhang et al., 2016); and Noonan-like syndrome with loose anagen hair after mutations in the SHOC2 or PPP1CB (Tajan et al., 2018). New gene research tools have led to a better understanding of the complexity of RAS signaling and consequently to an expansion of the pathogenic etiology of RASopathies. In this context, the genes identified that represent novel genes causative for RASopathy include the RIT1, SOS2, RASA2, RRAS, and SYNGAP1 genes (Tidyman \& Rauen, 2016). The RAS/MAPK pathway has been shown to be the predominant biochemical hallmark of the RASopathies. However, aberrant Ras signaling due to other effector pathways also appears to play an important role (Tidyman \& Rauen, 2016).

Piebaldism, Waardenburg syndrome and peripheral demyelinating neuropathy-central dysmyelinating- Waardenburg syndrome-Hirschsprung disease $(\mathrm{PCWH})$ are genetic disorders secondary to aberrant melanoblast migration during embryogenesis (Oiso et al., 2013). The binding of the KIT ligand (KITLG) to its KIT tyrosine kinase receptor, that triggers the Ras/mitogen-activated protein kinase signaling pathway, regulates melanocyte migration, differentiation and survival, as well as cell proliferation, melanogenesis and melanosome transfer (Oiso et al., 2013). Among these reported diseases, piebaldism (Zhang et al., 2016) and Waardenburg syndrome type 2E may present CALM as a clinical manifestation. Other disorders related to the KITLG / KIT signaling pathway that may also have CALM are familial progressive hyperpigmentation and hypopigmentation (FPHH) and familial progressive hyperpigmentation (FPH) (Oiso et al., 2013; Zhang et al., 2016).

Defects at any stage of neural crest cell development, such as migration, proliferation, cell-to-cell interaction, differentiation or growth, are associated with the pathophysiology of neurocutaneous syndrome or phakomatoses (Sarnat \& Flores-Sarnat, 2005; Gursoy \& Erçal, 2018). This group includes pathologies with different genetic mechanisms (Sarnat \& Flores-Sarnat, 2005; Klar, Cohen \& Lin, 2016). So we have neurofibromatosis type I that exhibits mutations in the NF1 gene, which encodes neurofibrin, a negative regulator of RAS signaling that is also expressed in migrating neural crest cells during early fetal development; neurofibromatosis type II, where mutations occur in the NF2 gene that encodes a 595 amino acid protein, named Merlin, a negative Schwann cell regulator whose impairment allows Schwann cells to proliferate excessively; tuberous sclerosis complex, where mutations in the TSC1 and TSC2 genes are responsible for the pathogenesis of the disease, leading to overactivation of the mTOR pathway, which plays an essential role in normal cell growth, proliferation, and survival (Gursoy \& Erçal, 2018). They usually involve inherited conditions, but spontaneous mutations can occur. As a common feature in the group, 
all diseases represent neurocristopathies and therefore share a common ectodermal embryologic origin, include abnormalities in the tissues of ectodermal origin, especially skin, eyes, and central nervous system (Reith, 2013). However, it is significant to emphasize that the neural crest is also important as an inducer of many tissues in craniofacial development and other mesodermal structures (Sarnat \& Flores-Sarnat, 2005). Neurofibromatosis type I, neurofibromatosis type II, tuberous sclerosis, ataxiatelangiectasia, Peutz-Jeghers syndrome, McCune-Albright syndrome and Cowden syndrome 1 are diseases belonging to this group that have CALM as a phenotypic manifestation.

In addition to these classifications, localized or general melanotic hyperpigmentation may be part of the clinical presentation of many other congenital systemic disorders that result from ubiquitous protein defects and/or basal cell processes (Baxter \& Pavan, 2013). This suggests that melanocytes are a cell type with high sensitivity to such disorders. A representative example is Fanconi anemia, a genetically heterogeneous disorder that affects DNA repair, characterized by different phenotypes that affect all organ systems (Baxter \& Pavan, 2013).

Among all characteristics of these hereditary syndromes associated with CALM, predisposition to tumors is one of the most important, considering the high levels of cancers associated with these syndromes, with many of these cancers presenting in childhood (Walsh et al., 2017). The incidence of specific types of cancer in the carriers of the germline mutations is dramatically high compared to the general population, considering that the rate of a simple somatic allelic loss is exponentially greater than the independent mutation of two alleles within the same cell (Elissen, 2016). Malignant tumors are the most common cause of death in individuals with some of these familial tumor syndromes (Brems, Beert, Ravel \& Legius, 2009).

The trend to develop tumors, which was observed in 16 of the 60 syndromes listed in this study, suggests a common underlying genetic basis. In this line, in neurofibromatosis type 1, the NFI acts as a tumor suppressor gene (Origone et al., 2003). In this condition, a germline pathogenic variant and a somatic mutation lead to homozygous inactivation of the $N F 1$ gene, resulting in a partial or total interruption of neurofibromin activity causing increased intracellular RAS signaling and abnormal cell proliferation (Origone et al., 2003). Different mechanisms are involved in the somatic inactivation observed in these hereditary syndromes, such as intragenic mutations (eg, nonsense, missense, frameshift, splice-site mutations, small insertions, and deletions), loss of heterozygosity, and hypermethylation of the promotor (Brems et al., 2009).

Another example is the genetically proven constitutional mismatch repair deficiency syndrome (CMMRD), a disease with multiple CALMs and other features of NF1 that are also part of the clinical findings. It is speculated that the remaining NF1 signals in patients with CMMRD result from post-zygotic mutations of the NF1 gene that may occur more frequently than normal in the population due to an accelerated rate of NF1 mutation in cells without a functional MMR system. However, it is also possible that CALM and other NF1 resources in these patients represent "isolated" skin manifestations (Maertens et al., 2007; Wimmer et al., 2017). Ataxia telangiectasia, Bloom syndrome, Nijmegen rupture syndrome and Fanconi anemia are among the most common DNA repair diseases and may present with CALM (Walsh et al., 2017). Pathogenic germline mutations in genes encoding proteins key in DNA repair and telomere biology result in the characteristic physical findings observed in patients with these hereditary disorders and in a high risk of cancer associated with these syndromes (Walsh et al., 2017).

\section{Conclusion}

The presence of CALM during a clinical evaluation of a patient should always be critically assessed by the healthcare professional, who must be aware of the possibility of an associated genetic syndrome. A detailed clinical evaluation should be performed by the physician to identify signs and symptoms that indicate the presence of any systemic disease. 
If a genetic syndrome is suspected, given the genetic complexity and phenotypic heterogeneity of diseases that present CALM, the large number of overlapping features in similar diseases, and the incurable nature of these conditions, advanced testing is needed to distinguish between these syndromes, to provide genetic counseling to families, establish the prognosis and available therapeutic measures, monitor the potential risk to prevent complications.

It is important to note that, although the vast majority of genetic syndromes meet the criteria for a rare disease, it is estimated that there are between 6,000 and 8,000 different types of rare diseases described, with consequently millions of people affected by these diseases worldwide. The specific diagnosis of the genetic syndrome that affects a given patient has a great impact on their life, both in terms of clinical guidance and early treatment, and in the emotional sense, as it provides some comfort by obtaining an explanation for their symptoms, in addition to the possibility of obtaining support from groups of people affected by the same disease. Thus, research that systematizes knowledge on a given topic and organizes clinical signs and symptoms into a coherent system, as done in this study, can provide valuable tools in the process of building clinical reasoning and can add value to clinical practice, contributing to improved clinical outcomes.

\section{References}

Alawi, F. (2019). Using rare diseases as teaching models to increase awareness. Oral Surgery, Oral Medicine, Oral Pathology and Oral Radiology, 128(2), 99100. DOI: $10.1016 /$ j.oooo.2019.04.015

Allanson, J. E., Upadhyaya, M., Watson, G. H., Partington, M., MacKenzie, A., Lahey, D., ... Harper, P., S. (1991). Watson syndrome: is it a subtype of type 1 neurofibromatosis? Journal of Medical Genetics, 28, 752-756. DOI: 10.1136/jmg.28.11.752

Amyere, M., Vogt, T., Hoo, J., Brandrup, F., Bygun, A., Boon, L., ... Vikkula, M. (2011). KITLG mutations cause familial progressive hyper- and hypopigmentation. Journal of Investigative Dermatology, 131(6), 1234-1239. DOI: 10.1038/jid.2011.29

Aoki, Y., Niihori, T., Inoue, S., \& Matsubara, Y. (2016). Recent advances in RASopathies. Journal of Human Genetics, 61(1), 33-39. DOI: 10.1038/jhg.2015.114

Arora, H., Chacon, A. H., Choudhary, S., McLeod, M. P., Meshkov, L., Nouri, K., ... Izakovic, J. (2014). Bloom syndrome. International Journal of Dermatology, 53(7), 798-802. DOI: 10.1111/ijd.12408

Baas, A. F., Gabbett, M., Rimac, M., Kansikas, M., Raphael, M., Nievelstein, R. A., ... Wimmer, K. (2013). Agenesis of the corpus callosum and gray matter heterotopia in three patients with constitutional mismatch repair deficiency syndrome. European Journal of Human Genetics, 21(1), 55-61. DOI: 10.1038/ejhg.2012.117

Baxter, L. L., \& Pavan, W. J. (2013). The etiology and molecular genetics of human pigmentation disorders. Wiley Interdisciplinary Reviews: Developmental Biology, 2(3), 379-392. DOI: 10.1002/wdev.72

Boquett, J. A., \& Ferreira, R. J. (2010). Aspectos biológicos e atividade tumorigência da família proto-oncogênica Ras. Semina: Ciências Biológicas e da Saúde, 31(2), 201-211. DOI:10.5433/1679-0359.2011v32n2p201

Brems, H., Beert, E., Ravel, T., \& Legius, E. (2009). Mechanisms in the pathogenesis of malignant tumors in neurofibromatosis type 1. The Lancet Oncology, 10(5), 508-515. DOI: 10.1016/S1470-2045(09)70033-6

Brems, H., Chmara, M., Sahbatou, M., Denayer, E., Taniguchi, K., Kato, R., ... Legius, E. (2007). Germline loss-of-function mutations in SPRED1 cause a neurofibromatosis 1-like phenotype. Nature Genetics, 39(9), 1120-1126. DOI: 10.1038/ng2113

Campen, R., Mankin, H., Louis, D. N., Hirano, M., \& MacCollin, M. (2001). Familial occurrence of adiposis dolorosa. Journal of the American Academy of Dermatology, 44(1), 132-136. DOI: 10.1067/mjd.2001.110872

Cao, H., Alrejaye, N., Klein, O. D., Goodwin, A. F., \& Oberol, S. (2017). A review of craniofacial and dental findings of the RASopathies. Orthodontics \& Craniofacial Research, 20(1), 32-38. DOI: 10.1111/ocr.12144

Chatten, J., \& Voorhess, M. L. (1967). Familial neuroblastoma. Report of a kindred with multiple disorders, including neuroblastomas in four siblings. The New England Journal of Medicine, 277(23), 1230-1236. DOI: 10.1056/NEJM196712072772304

Chiu, Y. E., Dugan, S., Basel, D., \& Siegel, D. H. (2013). Association of Piebaldism, multiple café-au-lait macules, and intertriginous freckling: clinical evidence of a commom pathway between KIT and sprout-related, ena/vasodilator-stimulated phosphoprotein homology-1 domain containing protein 1 (SPRED1). Pediatric Dermatology, 30(3), 379-382. DOI: 10.1111/j.1525-1470.2012.01858.x

Cichorek, M., Wachulska, M., Stasiewicz, A., \& Tymińska, A. (2013). Skin melanocytes: biology and development. Postepy Dermatologii Alergologii, 30(1), 30-41. DOI: $10.5114 /$ pdia.2013.33376

Digilio, M. C., Lepri, F., Baban, A., Dentici, M. L., Versacci, P., Capolino, R., ... Dallapiccola, B. (2011). RASophaties: Clinical Diagnosis in the First Year of Life. Molecular Syndromology 1(6), 282-289. DOI: 10.1159/000331266 
Elissen, W. L. (2016) Genética molecular do câncer. Latest revision: 2016 [Accessed: March 01, 2021]. Avaliable at: https://www.medicinanet.com.br > conteudos > acp-medicine

Evans, D. G. (2009). Neurofibromatosis type 2 (NF2): A clinical and molecular review. Orphanet Journal of Rare Diseases, 4, 16. DOI: 10.1186/1750-1172-416

Ferner, R. E. (2007). Neurofibromatosis 1 and neurofibromatosis 2: A twenty first century perspective. The Lancet Neurology, 6(4), 340-351. DOI: $10.1016 / \mathrm{S} 1474-4422(07) 70075-3$

Fistarol, S. K., \& Itin, P. H. (2010). Disorders of pigmentation. Journal der Deutschen Dermatologischen Gesellschaaft, 8(3), 187-201. DOI: 10.1111/j.16100387.2009.07137.x

Ghada, M. H., Abdel-Salam, M. D., Hana, H., Afifi, M. D., Maha, M., Eid, M. D., ... Kholoussi, N. (2011). Ectodermal Abnormalities in Patients with Kabuki Syndrome. Pediatric Dermatology, 28(5), 507-511. DOI: 10.1111/j.1525-1470.2011.01495.x

Giugliani, L., Vanzella, C., Zambrano, M. B., Donis, K. C., Wallau, T. K. W., \& Costa, F. M. (2019). Clinical research challenges in rare genetic diseases in Brazil - Review Article. Genetics and Molecular Biology, 42(1), 305-311. DOI: http://dx.doi.org/10.1590/1678-4685-GMB-2018-0174

Guimarães, P. B., Branco, A. A., Carvalho, E., Lima, F. E., Almeida, J. R., Santos, J. B., ... De Perreli, T. (2002). Síndrome de Cowden: relato de um caso. Anais Brasileiro de Dermatologia, 77(6), 711-720. DOI: 10.1590/S0365-05962002000600009

Gursoy, S., \& Erçal, D. (2018). Genetic Evaluation of Common Neurocutaneous Syndromes. Pediatric Neurology, 89, 3-10. DOI: 10.1016/j.pediatrneurol.2018.08.006

Halal, F., Genrak, M. H., Baillargeon, J., \& Lesage, R. (1982). Gastro-Cutaneous Syndrome: Peptic Ulcer/Hiatal Hernia, Multiple Lentigines/Café-au-lait Spots, Hypertelorism, and Myopia. American Journal of Medical Genetics, 11, 161-176. DOI: 10.1007/s00105-019-4416-6

Hamm, H., Emmerich, K., \& Olk, J. (2019). Pigmentierte Flecken als mögliche Frühzeichen genetischer Syndrome. Der Hautarzt, 70, 506-513. DOI: $10.1007 / \mathrm{s} 00105-019-4416-6$

Hatipoglu, N., Kurtoglu, S., Kendirci, M., Keskin, M., \& Per, H. (2010). Neurofibromatosis Type 1 with Overlap Turner Syndrome and Klinefelter Syndrome. Journal of Tropical Pediatrics, 56(1), 69-72. DOI: 10.1093/tropej/fmp053

Hennekam, R. C. M., Allanson, J. E., Krantz, I. D., \& Gorlin, R. J. (2010). Gorlin's syndromes of the head and neck (5th ed). New York, NY: Oxford.

Jansen, S., Hoischen, A., Coe, B. P., Carvill, G. L., Van Esch, H., Bosch, D., ... Vries, B. B. A. (2018). A genotype-first approach identifies an intellectual disability-overweight syndrome caused by PHIP haploinsufficiency. European journal of human genetics, 26(1), 54-63. DOI: 10.1038/s41431-017-0039-5

Klar, N., Cohen, B., \& Lin, D. D. M. (2015). Neurocutaneous syndromes. Handbook of Clinical Neurology,135, 565-589. DOI: 10.1016/s0031-3955(16)383675

Madson, J. G. (2012). Multiple or familial café-au-lait spots is neurofibromatosis type 6: clarification of a diagnosis. Dermatology Online Journal,18(5), 4. DOI: $10.5070 / \mathrm{D} 33 \mathrm{~d} 56 \mathrm{c} 2 \mathrm{q} 8$

Maertens, O., De Schepper, S., Vandesompele, J., Brems, H., Heynes, I., Janssens, S., ... Messiaen, L. (2007). Molecular dissection of isolated disease features in mosaic neurofibromatosis type 1. The American Journal of Human Genetics, 81(2), 243-251. DOI: 10.1086/519562

Martelli, H., Jr. (2019). In reply to: Alawi F. "Using rare diseases as teaching models to increase awareness". Letter to the editor. Oral Surgery, Oral Medicine, Oral Pathology and Oral Radiology, 128, 690-691. DOI: 10.1016/j.000o.2019.07.002

Mertens, D. E. (2015). Research and evaluation in education and psychology: integrating diversity with quantitative, qualitative and mixed methods (5th ed).

Chapter 3: Literature Review and Focusing the Research. Thousand Oaks, CA: Sage Publication, pp. 89-121

Mulchandani, S., Bhoj, E. J., Luo, M., Powell-Hamilton, N., Jenny, K., \& Gripp, K. W. (2016). Maternal uniparental disomy of chromosome 20: a novel imprinting disorder of growth failure. Genetics in Medicine, 18, 309-315. DOI: 10.1038/gim.2015.103

Nishimura, G., Hasegawa, T., Fujino, M., Hori, N., \& Tomita, Y. (2003). Microcephalic osteodysplastic primordial short stature type II with cafe-au-lait spots and moyamoya disease. American Journal of Medical Genetics, 117(3), 299-301. DOI: 10.1002/ajmg.a.10230

Nyström, A. M., Ekvall, S., Stromberg, B., Holmstrom, G., Thuresson, A. C., Annerén, G., ... Bondeson, M. L. (2009). A severe form of Noonan syndrome and autosomal dominant café-au-lait spots-evidence for different genetic origins. Acta Paediatrica, 98, 693-698. DOI: 10.1111/j.1651-2227.2008.01170.X

Oates, J. A., \& Harris, R. L. (2015). Literature Review and Focusing the Research (Chapter 3). In R. Harris \& F. Williams (Eds.), Research and Evaluation in Education and Psychology, integrating diversity with quantitative, qualitative and mixed methods (5th ed). Thousand Oaks, CA: Sage Publication, pp. 89121.

Oiso, N., Fukai, K., Kawada, A., \& Suzuki, T. (2013). Piebaldism. The Journal of Dermatology, 40(5): 330-335. DOI: 10.1111/j.1346-8138.2012.01583.x

Online Mendelian Inheritance in Man, OMIM®: authoritative compendium of human genes and genetic phenotypes. McKusick-Nathans Institute of Genetic Medicine, Johns Hopkins University (Baltimore, MD). Available at: https://omim.org/

Origone, P., Defferrari, R., Mazzocco, K., Cunsolo, C. L., Bernardi, B., Tonini, G. P. (2003). Homozygous Inactivation of NF1 Gene in a Patient with Familial NF1 and Disseminated Neuroblastoma. American Journal of Medical Genetics, 118A(4), 309-313. DOI:10.1002/ajmg.a.10167

Orphanet: an online database of rare diseases and orphan drugs. Copyright, INSERM 1997. Available at: www.orpha.net 
Pagnamenta, A. T., Murray, J. E., Yoon, G., Sadighi Akha, E., Harrison, V., Bicknell, L. S., ... Knight, S. J. (2012). A novel nonsense CDK5RAP2 mutation in a Somali child with primary microcephaly and sensorineural hearing loss. American Journal of Medical Genetics, 158A, 2577-2582. DOI: $10.1002 /$ ajmg.a.35558

Picardo, M., \& Cardinali, G. (2011). The genetic determination of skin pigmentation: KITLG and the KITLG/c-Kit pathway as key players in the onset of human familial pigmentary diseases. Journal of Investigate Dermatology, 131(6), 1182-1185. DOI: 10.1038/jid.2011.67

Plana-Pla, A., Bielsa-Marsol, I., \& Carrato-Monino, C. (2017). Diagnostic and Prognostic Relevant of the Cutaneous Manifestations of Neurofibromatosis Type 2. Actas Dermo-Sifiliográficas, 108(7), 630-636. DOI: 10.1016/j.adengl.2016.12.025

Plataras, C., Christianakis, E., Fostira, F., Bourikis, G., Chorti, M., Bourikas, D., ... Eirekat, K. (2018). Asymptomatic Gastric Giant Polyp in a Boy with PeutzJeghers Syndrome Presented with Multiple Café Au Lait Traits. Case Reports in Surgery, 2018, 1-3, Article ID 6895974. DOI: 10.1155/2018/6895974

Reith, W. (2013). Phakomatosen. Der Radiologe, 53, 1075-1076. DOI: 10.1007/s00117-013-2532-3

Rinaldi, B., Vaisfeld, A., Amarri, S., Baldo C, Gobbi G, Magini P, ... Crimi, M. (2017). Guideline recommendations for diagnosis and clinical management of Ring14 syndrome-first report of an ad hoc task force. Orphanet Journal of Rare Diseases, 12(1), 69. DOI: 10.1186/s13023-017-0606-4

Rivers, J. K., MacLennan, R., Kelly, J. W., Lews, A. E., Tate, B. J., Harrison, S., ... McCarthy, W. H. (1985). The eastern Australian childhood nevus study: prevalence of atypical nevi, congenital nevus-like nevi, and other pigmented lesions. Journal of the American Academy of Dermatology, 32(6), 957-963. DOI: $10.1016 / 0190-9622(95) 91331-9$

Ronnstrand, L. (2004). Signal transduction via the stem cell factor receptor/c-Kit. Cellular and Molecular Life Sciences, 61(19-20), 2535-2548. DOI: $10.1007 / \mathrm{s} 00018-004-4189-6$

Rosales-Solis, G. M., Martínez-Longoria, C. A., Guerrero-González, G. A., Ocampo-Garza, J., \& Ocampo-Candiani, J. (2016). Síndrome de Bloom. Manifestaciones clínicas y estudio cromosómico en una niña mexicana [Bloom syndrome. Clinical manifestations and cromosomal study in a Mexican child]. Gaceta medica de Mexico, 152(6), 836-837. PMID: 27861482

Sarkozy, A., Digilio, M. C., \& Dallapiccola, B. (2008). Leopard Syndrome. Orphanet Journal of Rare Diseases, 3, 13. DOI: 10.1186/1750-1172-3-13 Sarnat, H. B., \& Flores-Sarnat, L. (2005). Embryology of the neural crest: its inductive role in the neurocutaneous syndromes. Journal of Child Neurology, 20(8), 637-643. DOI: 10.1177/08830738050200080101

Shah, K. N. (2010). The Diagnostic and Clinical Significance of Café-au-lait Macules. Pediatric Clinics of North America, 57(5), 1131-1153. DOI: 10.1016/j.pcl.2010.07.002

Spiteri, B. S., Stafrace, Y., Calleja-Agius J. (2017). Silver-Russell Syndrome: A Review. Neonatal Network, 36(4), 206-212. DOI: 10.1891/0730-0832.36.4.206.

Tajan, M., Paccoud, R., Branks, S., Edouard, T., \& Yart, A. (2018). The RASopathy Family: Consequences of Germline Activation of the RAS/MAPK Pathway. Endocrine Reviews, 39(5), 676-700. DOI: 10.1210/er.2017-00232

Tidyman, W. E., \& Rauen, K. A. (2016). Expansion of the RASopathies. Current Genetic Medicine Reports, 4(3): 57-64. DOI: 10.1007/s40142-016-0100-7

Veenma, D. C. M., Eussen, H. J., Govaerts, L. C. P., de Kort, S. W. K., Odink, R. J., Wouters, C. H., ... de Klein, A. (2010). Phenotype-genotype correlation in a familial IGF1R microdeletion case. Journal of Medical Genetics, 47, 492-498. DOI: 10.1136/jmg.2009.070730.

Walsh, M. F., Chang, V. Y., Kohlmann, W. K., Scott, H. S., Cunniff, C., Bourdeaut, F., ... Savage, S. A. (2017). Recommendations for Childhood Cancer Screening and Surveillance in DNA Repair Disorders. Clinical Cancer Research, 23(11), 23-31. DOI: 10.1158/1078-0432.CCR-17-0465

Wimmer, K., Rosenbaum, T., \& Messiaen, L. (2017). Connections between constitucional mismatch repair deficienty syndrome and neurofibromatosis type 1. Clinical genetics, 91(4), 507-519. DOI: 10.1111/cge.12904

Young I. D., Barrow, M., \& Hall, C. M. (2004). Microcephalic Osteodysplastic Primordial Short Stature Type II with Café-au-lait Spots and Moyamoya Disease: Another Patient. American Journal of Medical Genetics, 127(A), 218-220. DOI: 10.1002/ajmg.a.20647

Zeng, L., Zheng, X. D., Liu L. H., Fu, L. Y., Zuo, X. B., Chen, G., ... Zhang, X. J. (2016). Familial progressive hyperpigmentation and hypopigmentation without KITLG mutation. Clinical and Experimental Dermatology, 41(8), 927-929. DOI: 10.1111/ced.12923

Zhang, J., Li, M., \& Yao, Z. (2016). Molecular screening strategies for NF1-like syndromes with café-au-lait macules (Review). Molecular Medicine Reports, 14(5), 4023-4029. DOI: 10.3892/mmr.2016.5760 